\title{
Amorphous microwires with enhanced magnetic softness and GMI characteristics
}

\author{
A. Zhukova1,2, M. Ipatov ${ }^{2}$ and V. Zhukova ${ }^{2}$ \\ ${ }^{1}$ IKERBASQUE, Basque Foundation for Science, 48011 Bilbao, Spain \\ ${ }^{2}$ Dpto. Fisica de Materiales, Fac. Quimicas, UPV/EHU, 20009 San Sebastian, Spain
}

\begin{abstract}
In this paper we present results on correlation of GMI effect and soft magnetic behavior in Co-rich microwires with low magnetostriction constant. Correlation between magnetoelastic anisotropy and magnetic field dependences of diagonal and off-diagonal impedance components are observed. Low field GMI hysteresis, explained in terms of magnetoelastic anisotropy of microwires, has been suppressed by the bias current.
\end{abstract}

\section{Introduction}

Magnetically soft glass coated microwires (typically of 5-30 $\mu \mathrm{m}$ in diameter) exhibit a number of outstanding magnetic properties such as magnetic bistability and giant magneto-impedance, GMI, effect [1, 2]. Recently, excellent soft magnetic properties and GMI effect of glass coated microwires attracted great attention [3, 4], giving rise to development of industrial applications for low magnetic field detection [5].

Giant magneto-impedance effect (GMI), consisting of large sensitivity of the impedance of magnetically soft conductor on applied magnetic field, attracted great attention in the field of applied magnetism [3-7] especially because of excellent magnetic field sensitivity suitable for low magnetic field detection. Such GMI effect is especially high in ferromagnetic magnetically soft wires (especially of amorphous and nanocrystalline origin) [3, 6, 7]. It is worth mentioning, that the cylindrical shape is quite suitable for achieving of high GMI effect [3, 6, 7]. General tendency on miniaturization of magnetic sensors requires development of thin soft magnetic materials, like thin wires and thin films [1,5]. Owing to its thin dimensions, glass-coated microwires gained special interest in the filed of applied magnetism for designing of the sensors based on GMI effect $[3,4]$.

It is worth mentioning, that in most of applications a high linearity of MI dependence and low hysteresis are desirable [7, 8]. Anti-symmetrical MI curve with linear region has been obtained in current pulsed excitation scheme of wires using detection of off-diagonal GMI component $[3,8$, and 9]. Such pulsed scheme for GMI measurements resulted quite useful for real GMI sensors development [5]. At the same time we have recently showed, that linearity and shape of off-diagonal component in microwires can be tailored by thermal treatment [10]. Considerable GMI hysteresis has been observed and analyzed in microwires possessing helical anisotropy [9], although enhanced magnetic field sensitivity of the GMI effect in amorphous wires is related to specific outer domain structure in the surface area [11].

\footnotetext{
a e-mail : arkadi.joukov@ehu.es; wupzhuka@sp.ehu.es
}

This is an Open Access article distributed under the terms of the Creative Commons Attribution License 2.0, which permits unrestricted use, distribution, and reproduction in any medium, provided the original work is properly cited. 
In this paper we studied the GMI effect (GMI ratio, $\Delta Z / Z$, diagonal $Z_{z z}$ and off-diagonal impedance tensor $\zeta_{\phi z}$ components) and hysteretic magnetic properties in ultra-thin amorphous glass-coated microwires with vanishing magnetostriction constant.

\section{Experimental details}

We have measured dependences of the diagonal $Z_{z z}$ and off-diagonal $Z_{\varphi_{Z}}$ impedance components on external axial magnetic field $\mathrm{H}$ in Co-rich microwires, as described elsewhere [3, 8, 9]. The microwires with nominal compositions $\mathrm{Co}_{67.1} \mathrm{Fe}_{3.8} \mathrm{Ni}_{1.4} \mathrm{Si}_{14.5} \mathrm{~B}_{11.5} \mathrm{Mo}_{1.7}$ and $\mathrm{Co}_{66} \mathrm{Cr}_{3.5} \mathrm{Fe}_{3.5} \mathrm{~B}_{16} \mathrm{Si}_{11}$ different diameters of metallic nucleus, $d$, total diameters, $D$, and consequently different $\rho$ - ratios $(\rho=d / D)$ have been fabricated by the Taylor-Ulitovsky method $[3,10]$.

The microwires were placed in a specially designed microstrip cell. One wire end was connected to the inner conductor of a coaxial line through a matched microstrip line while the other was connected to the ground plane. The components $Z_{z z}$ and $Z_{\varphi_{z}}$ were measured simultaneously using vector network analyzer. The diagonal impedance of the sample $Z w=Z z z l$, where $l$ is the wire length, was obtained from reflection coefficient $S_{11}$ and the off-diagonal impedance $Z_{\varphi_{z}}$ was measured as transmission coefficient $S_{21}$ as a voltage induced in a 2-mm long pick-up coil wounded over the wire. The static bias field $H_{B}$ was created by the dc current $I_{B}$ applied to sample through the bias-tee element. The other experimental details are given in Ref. 8. The frequency range for the offdiagonal component $Z_{\varphi_{z}}$ was $10-300 \mathrm{MHz}$, while diagonal impedance component has been measured till $7 \mathrm{GHz}$.

For practical sensor it is essential to have the anti-symmetrical dependence on magnetic field, so off-diagonal MI components could be more suitable. In the sensor application, pulse excitation is preferred over sinusoidal because of simple electronic design and low power consumption, therefore we used also pulsed excitation scheme, as described elsewhere $[3,9]$.

Hysteresis loops have been measured by the induction method, as described elsewhere [3, 10].

\section{Results and discussion}

Magnetic field, $\mathrm{H}$, dependence of real part, $Z_{1}$ of the longitudinal wire impedance $\mathrm{Z}_{\mathrm{zz}}\left(\mathrm{Z}_{\mathrm{zz}}=\mathrm{Z}_{1}+i Z_{2}\right)$, measured till $4 \mathrm{GHz}$ in $\mathrm{Co}_{66} \mathrm{Cr}_{3.5} \mathrm{Fe}_{3.5} \mathrm{~B}_{16} \mathrm{Si}_{11}$ microwire is shown in Fig.1. General features of these dependences is that the magnetic field of maximum shifts to the higher field region increasing the $f$. High enough magnetic field sensitivity, i.e. GMI effect till GHz- range frequencies should be also underlined.
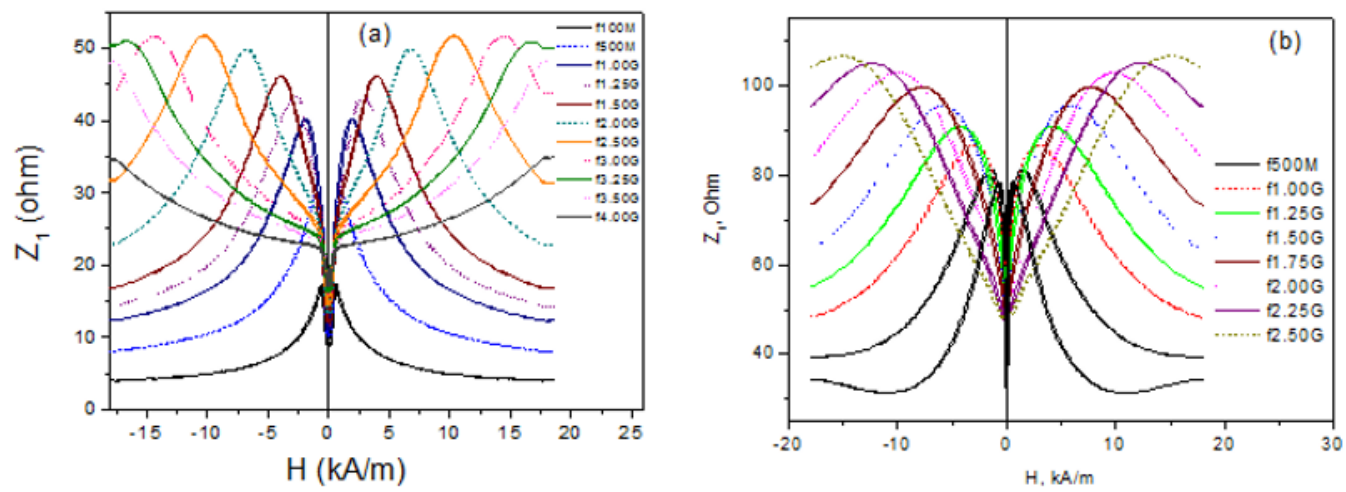

Fig.1. $\mathrm{Z}(\mathrm{H})$ dependence of $\mathrm{Co}_{66} \mathrm{Cr}_{3.5} \mathrm{Fe}_{3.5} \mathrm{~B}_{16} \mathrm{Si}_{11}$ (a) and $\mathrm{Co}_{67} \mathrm{Fe}_{3.85} \mathrm{Ni}_{1.45} \mathrm{~B}_{11.5} \mathrm{Si}_{14.5} \mathrm{Mo}_{1.7}$ (b) microwires measured at different frequencies 
Off-diagonal and low field diagonal components of GMI, measured in $\mathrm{Co}_{67} \mathrm{Fe}_{3.85} \mathrm{Ni}_{1.45} \mathrm{~B}_{11.5} \mathrm{Si}_{14.5} \mathrm{Mo}_{1.7}$ microwire are shown in Fig.2. Important feature of Fig.2 is a considerable hysteresis for both off-diagonal and longitudinal impedance.
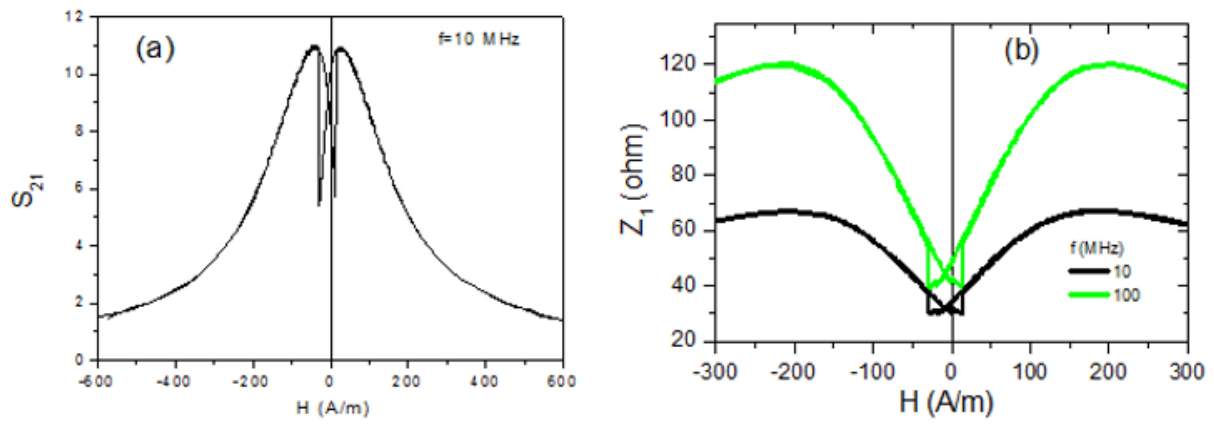

Fig. 2. Magnetic field dependences of the coefficient $S_{21}$ at $10 \mathrm{MHz}$ (a) and $\mathrm{Z}_{1}(\mathrm{H})$ dependences at different frequencies (b) measured in $\mathrm{Co}_{66} \mathrm{Cr}_{3.5} \mathrm{Fe}_{3.5} \mathrm{~B}_{16} \mathrm{Si}_{11}$ microwire

Fig. 3 shows field dependence of the off-diagonal voltage response, $V_{\text {out }}$ measured using pulsed scheme, described elsewhere $[3,9,12,13]$ in $\mathrm{Co}_{67.1} \mathrm{Fe}_{3.8} \mathrm{Ni}_{1.4} \mathrm{Si}_{14.5} \mathrm{~B}_{11.5} \mathrm{Mo}_{1.7}\left(\lambda_{s} \approx 3 \cdot 10^{-7}\right)$ microwire with different geometry: metallic nucleus diameter and total diameter with glass coating are 6.0/10.2 $(\rho \approx 0,59), 7.0 / 11.0 \quad(\rho \approx 0,64)$ and 8.2/13.7 $\mu \mathrm{m}(\rho \approx 0,6)$. The off-diagonal components exhibit antisymmetrical magnetic field dependence, suitable for determination the magnetic field direction in real sensor devices $[3,9,12,13]$. It should be noted from Fig.3 that the $V_{\text {out }}(H)$ curves exhibit nearly linear growth within the field range from $-H_{m}$ to $H_{m}$. The $H_{m}$ limits the working range of MI sensor to $240 \mathrm{~A} / \mathrm{m}$ and should be associated with the anisotropy field. The effect of the $\rho-$ ratio on $\mathrm{V}_{\text {out }}(\mathrm{H})$ (Fig.3) should be attributed to the effect of internal stresses on the magnetic anisotropy field. It must be underlined, that all studied samples exhibited excellent magnetically soft properties with inclined hysteresis hoops and extremely low coercivities (between 4 and 10A/m). Magnetic anisotropy field, $H_{k}$, is found to be determined by the $\rho$-ratio, decreasing with $\rho$ (Fig.4), as also has been reported earlier $[3,10]$.

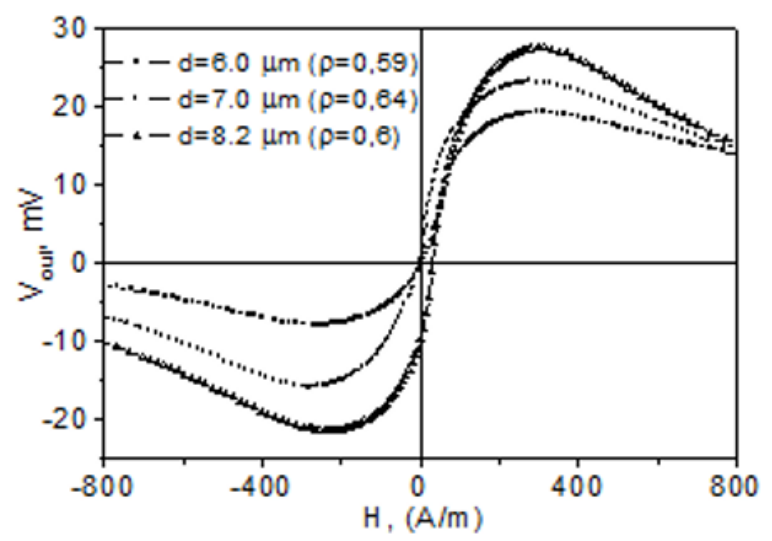

Fig.3. $\mathrm{V}_{\text {out }}(\mathrm{H})$ response of $\mathrm{Co}_{67,1} \mathrm{Fe}_{3,8} \mathrm{Ni}_{1,4} \mathrm{Si}_{14,5} \mathrm{~B}_{11,5} \mathrm{Mo}_{1,7}$ microwires with different diameters, d, and $\rho$ ratios

On the other hand, it is well established, that strength of internal stresses, $\sigma_{i}$, arising during simultaneous rapid quenching of metallic nucleus surrounding by the glass coating can be controlled by the $\rho$-ratio: strength of internal stresses increases decreasing $\rho$-ratio (i.e. increases with 
increasing of the glass volume). Consequently magnetic field dependences of both $Z_{z z}$ and $Z_{\varphi_{z}}$ can be controlled by $\rho$-ratio.
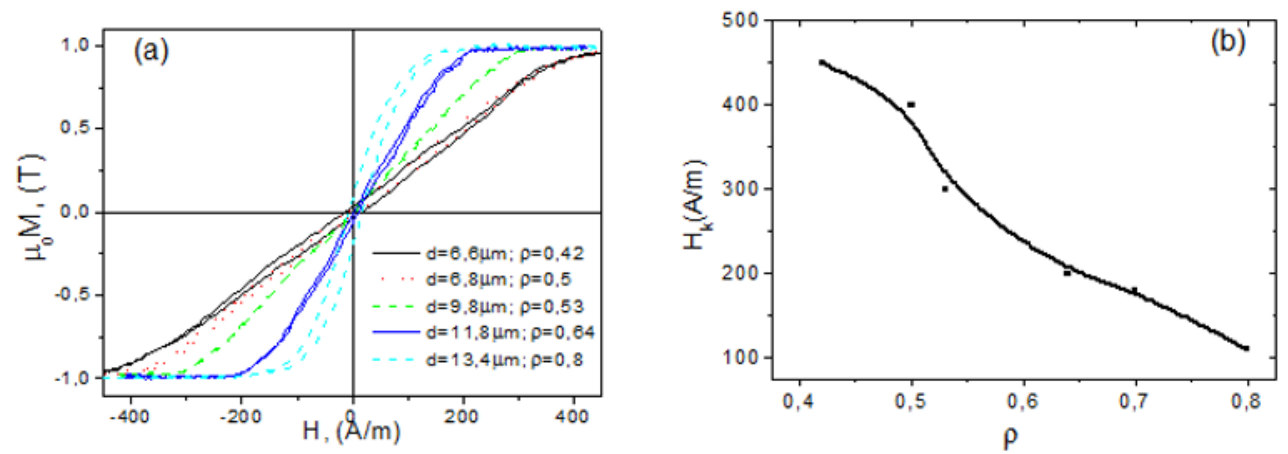

Fig.4. Hysteresis loops of $\mathrm{Co}_{67.1} \mathrm{Fe}_{3.8} \mathrm{Ni}_{1.4} \mathrm{Si}_{14.5} \mathrm{~B}_{11.5} \mathrm{Mo}_{1.7}$ microwires with different geometry (a) and dependence of $\mathrm{H}_{\mathrm{k}}$ on $\rho$-ratio (b).

Additionally, we recently found, that the nature of observed low field hysteresis on $\mathrm{Z}_{1}(\mathrm{H})$ and $Z_{\varphi_{z}}$ $(\mathrm{H})$ is directly related with deviation of the anisotropy easy axis from transversal direction [8]. Therefore, application of circular bias magnetic field $H_{B}$ produced by DC current $I_{B}$ running through the wire affects the hysteresis and asymmetry of the MI dependence, suppressing this hysteresis when $I_{B}$ is high enough (see Fig.5, where effect of bias voltage on diagonal impedance, $Z_{1}$, and on $\mathrm{S}_{21}$ parameter, proportional to off-diagonal GMI component is shown). In fact in pulsed exciting scheme when the sharp pulses with pulse edge time about $5 \mathrm{~ns}$ are produced by passing square wave multi-vibrator pulses through the differentiating circuit, overall pulsed current contains a DC component that produces bias circular magnetic field $[6,7]$. In this way low field hysteresis can be surpassed selecting adequate pulse amplitude.
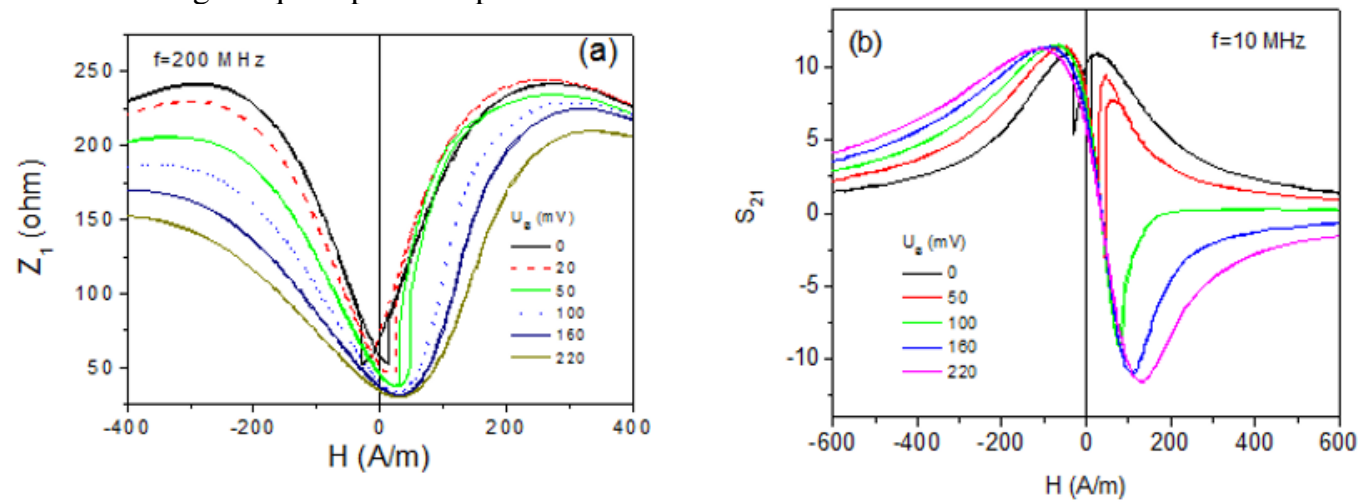

Fig.5. Effect of bias voltage $U_{B}$ on magnetic field dependence of diagonal impedance (a) and $S_{21}$ parameter (b) of $\mathrm{Co}_{67} \mathrm{Fe}_{3.85} \mathrm{Ni}_{1.45} \mathrm{~B}_{11.5} \mathrm{Si}_{14.5} \mathrm{Mo}_{1.7}$ microwire

Traditional way to tailor magnetoelastic anisotropy issue of thermal treatment. The influence of Joule heating on off-diagonal field characteristic of nearly zero magnetostriction $\mathrm{Co}_{67.1} \mathrm{Fe}_{3.8} \mathrm{Ni}_{1.4} \mathrm{Si}_{14.5} \mathrm{~B}_{11.5} \mathrm{Mo}_{1.7}$ microwire with diameters 9.4/17.0 $\mu \mathrm{m}(\rho \approx 0,55)$ is shown in Fig. 6. One can see that the thermal annealing with $50 \mathrm{~mA} \mathrm{DC}$ current reduces the $\mathrm{H}_{\mathrm{m}}$ from $480 \mathrm{~A} / \mathrm{m}$ in ascast state to $240 \mathrm{~A} / \mathrm{m}$ after 5 min annealing.

Observed $H_{k}(\rho)$ dependence has been attributed to the magnetoelastic energy contribution given by

$$
K_{m e} \approx 3 / 2 \lambda_{s} \sigma_{l},
$$



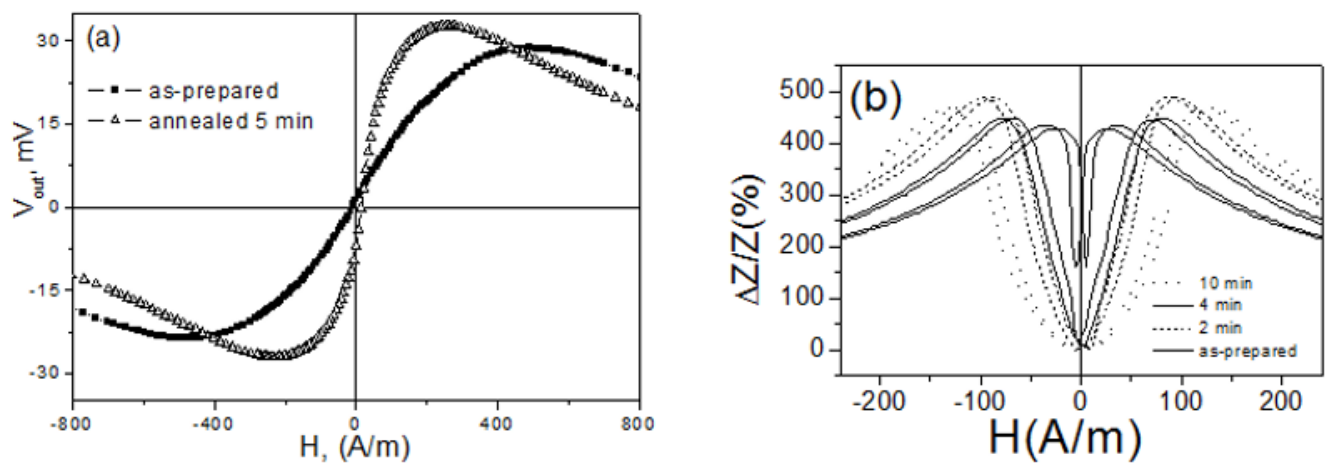

Fig.6. $V_{\text {out }}(H)$ of as -prepared and Joule-heated $\mathrm{Co}_{67} \mathrm{Fe}_{3.85} \mathrm{Ni}_{1.45} \mathrm{~B}_{11.5} \mathrm{Si}_{14.5} \mathrm{Mo}_{1.7}$ microwire (current annealing with $50 \mathrm{~mA}$ current intensity) (a) and $\Delta Z / Z(H)$ dependences of heated $\mathrm{Co}_{67} \mathrm{Fe}_{3.85} \mathrm{Ni}_{1.45}$

$\mathrm{B}_{11.5} \mathrm{Si}_{14.5} \mathrm{Mo}_{1.7}$ microwire measured at $\mathrm{f}=30 \mathrm{MHz}$ and $\mathrm{I}=1 \mathrm{~mA}$ in microwire subjected to $\mathrm{CA}$ annealing at $40 \mathrm{~mA}$ for different time (b)

where $\lambda_{s}$ is the saturation magnetostriction and $\sigma_{i}$ is the internal stress. The magnetostriction constant is mostly determined by the chemical composition and achieves almost nearly-zero values in amorphous alloys based on Fe-Co with $\mathrm{Co} / \mathrm{Fe} \approx 70 / 5 \lambda_{s} \approx 0[3,14,15]$. On the other hand, the estimated values of the internal stresses in these glass coated microwires arising from the difference in the thermal expansion coefficients of simultaneously solidifying metallic nucleus and glass coating are of the order of 100-1000 MPa, depending strongly on the ratio between the glass coating thickness and metallic core diameter [3, 15-17], increasing with decreasing $\rho$-ratio. Consequently, magnetoelastic anisotropy of glass-coated microwires can be controlled by the geometrical ratio $\rho$ through the strength of internal stresses.

Application of stress and/or magnetic filed during annealing of microwires allows inducing considerable magnetic anisotropy and results in some cases in drastic changes of hysteretic magnetic properties and GMI behavior [10, 12, 18]. As an example, application of axial magnetic field during annealing induces axial magnetic anisotropy in Co-rich microwires (Fig.7). Here hysteresis loops of $\mathrm{Co}_{67} \mathrm{Fe}_{3.85} \mathrm{Ni}_{1.45} \mathrm{~B}_{11.5} \mathrm{Si}_{14.5} \mathrm{Mo}_{1.7}$ microwires $(\mathrm{d}=22.4 \mu \mathrm{m}, \mathrm{D}=22.8 \mu \mathrm{m})$ annealed by Joule heating without (CA) and under application of axial magnetic field (FCA) are shown. As can be appreciated, increasing of remanent magnetization and decreasing of coercivity after FCA is observed.

Most significant changes of both hysteresis loop and GMI behaviour have been observed in Fe-rich microwires subjected to stress annealing (Fig.7).
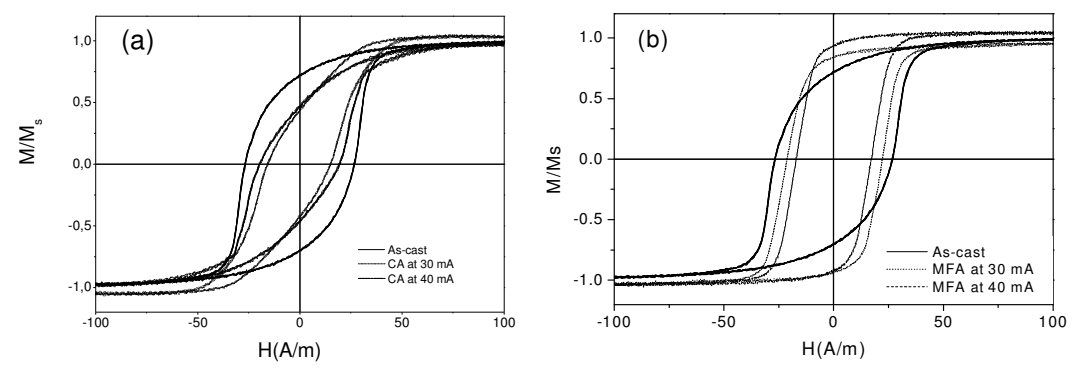

Fig.7. Effect of CA (a) and FCA (b) treatments on bulk hysteresis loops of $\mathrm{Co}_{67} \mathrm{Fe}_{3.85} \mathrm{Ni}_{1.45} \mathrm{~B}_{11.5} \mathrm{Si}_{14.5} \mathrm{Mo}_{1.7}$ microwires (d=22.4 $\left.\mu \mathrm{m}, \mathrm{D}=22.8 \mu \mathrm{m}\right)$. 
Stress annealing of $\mathrm{Fe}_{74} \mathrm{~B}_{13} \mathrm{Si}_{11} \mathrm{C}_{2}$ microwires resulted in induction of considerable stress induced anisotropy [18]. The shape of hysteresis loop depends on time and temperature of annealing (Fig.8a). In this case the easy axis of magnetic anisotropy has been changed from axial to transversal [18]. Additionally, application of stress to annealed microwires with well-defined transverse anisotropy results in drastic change of the hysteresis loop (Fig.8b).

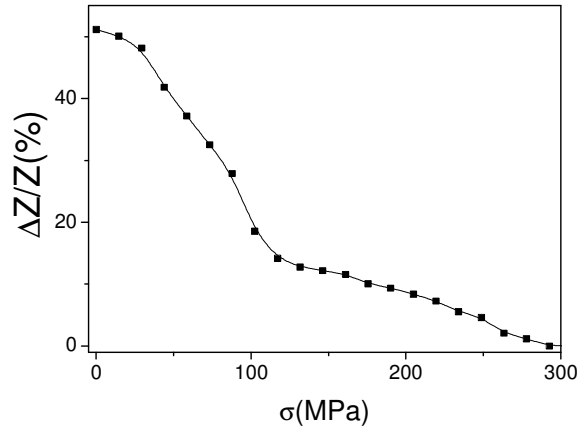

Fig.8. Stress impedance effect of stress annealed $\mathrm{Fe}_{74} \mathrm{~B}_{13} \mathrm{Si}_{11} \mathrm{C}_{2}$ glass-coated microwire under stress (468 $\mathrm{MPa}$ ) at $275^{\circ} \mathrm{C}$ for $0.5 \mathrm{~h}$ measured at frequency, $f=10 \mathrm{MHz}$ for the driving current amplitude of $2 \mathrm{~mA}$

Origin of such stress-induced anisotropy is related with so-called "Back stresses" originated from the composite origin of glass-coated microwires annealed under tensile stress: compressive stresses compensate axial stress component and under these conditions transversal stress components are predominant [18].

Consequently, these stress annealed samples exhibit stress-impedance effect, i.e. impedance change $(\Delta Z / Z)$ under applied stress, $\sigma$, observed in samples with stress induced transversal anisotropy (see Fig.9) [18,19].

It should be assumed that the internal stresses relaxation after heat treatment should drastically change both the soft magnetic behavior and $\Delta Z / Z(H)$ dependence due to stress relaxation, induced magnetic anisotropy and change of magnetostriction constant under annealing.

Summarizing, a number of interesting phenomena can be observed in thin magnetically soft ferromagnetic microwires. Taylor Ulitovky technique allows fabrication of composite microwires with thin metallic nucleus diameter. Composite character of such microwires results in appearance of additional magnetoelastic anisotropy. Heat treatment is the efficient method of tailoring of magnetic properties and GMI effect of such microwires. Selection of proper chemical composition, geometry and adequate conditions of annealing allows achieving of high GMI effect.

\section{Conclusions}

In thin amorphous wires, produced by the Taylor-Ulitovsky technique, magnetic softness and magnetic field dependence of GMI effect (both longitudinal and off-diagonal) and GMI hysteresis are determined the magnetoelastic anisotropy. This magnetoelastic anisotropy can be tailored by the sample geometry and adequate annealing. There are a number of interesting effects, such as induction of the transversal anisotropy in Fe-rich microwires allowing creating extremely stress sensitive elements. Studies of diagonal and off-diagonal MI tensor components of glass-coated microwires have shown the grate potential of these materials for microminiaturized magnetic field sensor application. Their main advantages are high sensitive low-hysteresis field dependence. By varying the alloys composition and applying post fabrication processing it is possible to control the sensor's operating range. Low field GMI hysteresis has been observed and explained in terms of helical magnetic anisotropy of microwires. 


\section{Acknowledgment}

This work was supported by EU ERA-NET programme under projects "DEVMAGMIWIRTEC" (MANUNET-2007-Basque-3) and "SoMaMicSens" (MANUNET-2010-Basque-3), by EU under FP7 "EM-safety" project, by Spanish Ministry of Science and Innovation, MICINN under Project MAT2010-18914 and by the Basque Government under Saiotek 10 MIMAGURA project (SPE11UN087)

\section{References}

1. D. C. Jiles, Acta Materialia,. 51, 5907 (2003).

2. A. P. Zhukov, Materials and Design, 5, 299 (1993)

3. V. Zhukova, M. Ipatov and A Zhukov, Sensors 9, 9216 (2009)

4. A. F. Cobeño, A. Zhukov, J. M. Blanco and J. Gonzalez, J. Magn. Magn. Mat 234, L359 (2001)

5. K. Mohri and Y. Honkura Sensors letters, 5 , 267 (2007)

6. $\quad$ L. V. Panina and K. Mohri, Appl. Phys. Lett. 65 1189, (1994).

7. $\quad$ R. Beach and A. Berkowitz, Appl. Phys. Lett. 643652 (1994)

8. M. Ipatov, V. Zhukova, A. Zhukov, J. Gonzalez, and A. Zvezdin, Phys. Rev. B 8134421 (2010)

9. S. I. Sandacci, D. P. Makhnovskiy, L. V. Panina, K. Mohri, and Y. Honkura, IEEE Trans Magn., 353505 (2004).

10. A. Zhukov, M. Ipatov, J. Gonzalez, J. M. Blanco, V. Zhukova, J. Magn. Magn. Mater. 321822 (2009)

11. V. Zhukova, N. A. Usov, A. Zhukov and J. Gonzalez, Phys. Rev B, 65 134407-1 (2002)

12. A. Zhukov, M. Ipatov and V. Zhukova, Journal of Physics: Conference Series 303012085 (2011)

13. A. Zhukov, M. Ipatov, J. M. Blanco, J. Gonzalez, and V. Zhukova, Phys. Status Solidi A 206, No. 4, 674 (2009)

14. H. Fujimori, K. I. Arai, H. Shirae, H. Saito, T. Masumoto and N. Tsuya, Japan. J. Appl. Phys. 15 705 (1976)

15. A. Zhukov and V. Zhukova, "Magnetic properties and applications of ferromagnetic microwires with amorphous and nanocrystalline structure", Nova Science Publishers, Inc. 400 Oser Avenue, Suite 1600 Hauppauge, NY 11788, 2009, 162 p. ISBN: 978-1-60741-770-5.

16. H. Chiriac, T. A. Ovari, and Gh. Pop, Phys. Rev. B 4210105 (1995)

17. H. Chiriac, T.-A- Ovari and A. Zhukov, J. Magn. Magn. Mater. 254-255, 469(2003)

18. A. Zhukov, Adv. Func. Mat., 16675 (2006)

19. M. Vazquez, H. Chiriac, A. Zhukov, L. Panina and T. Uchiyama, Phys. Status Solidi A, A 208, No. 3, (2011) 493 